\title{
Trichotillomania in Children and the Different Approaches between Dermatological and Behavioral Health Professionals at an Urban Tertiary Care Center
}

\author{
Sneha Rangu ${ }^{a}$ Leslie Castelo-Soccio ${ }^{a, b}$ \\ a Section of Dermatology, The Children's Hospital of Philadelphia, Philadelphia, PA, USA; bUniversity of Pennsylvania \\ Perelman School of Medicine, Philadelphia, PA, USA
}

\section{Keywords}

Trichotillomania $\cdot$ Trichotillosis $\cdot$ Hair pulling $\cdot$ Pediatric dermatology $\cdot$ Behavioral health

\begin{abstract}
Background: Trichotillomania (TTM) is a complex disease with varying clinical characteristics, and psychosocial impairment is noted in many TTM patients. Despite its prevalence in childhood, there is limited research on pediatric TTM. Objective: To analyze the clinical and epidemiologic features of TTM in children evaluated by dermatologists and behavioral health specialists. Method: We performed a retrospective chart review of 137 pediatric patients seen at the Children's Hospital of Philadelphia with initial presentation of TTM at age 17 or younger. Patients were treated by dermatology or behavioral health. Results: The majority of the patients were females, with an average diagnosis age around 8 years. Over half had a psychiatric comorbidity, and over a quarter had a skin disorder. Skin disorders were more commonly present in those evaluated by dermatology, and psychiatric comorbidities were more commonly present in those evaluated by behavioral health. The most common form of treatment was behavioral therapy, with medications
\end{abstract}

prescribed more often by dermatologists. Conclusions: TTM patients choose to present to behavioral health or dermatology; however, there are distinctive differences between the two cohorts. With behavioral and pharmacologic treatment options, a relationship between dermatologists and behavioral health specialists is necessary for multifactorial management of TTM.

(c) 2020 S. Karger AG, Basel

\section{Introduction}

The first case of trichotillomania (TTM), also known as trichotillosis, was reported in 1889 by a French dermatologist, Hallopeau [1]. It falls under the obsessive-compulsive and related disorders in the Diagnostic and Statistical Manual of Mental Disorders-V (DSM-V). It is assigned to individuals who recurrently pull out hair from any region of their body resulting in hair loss and other forms of impairment [2]. The most common sites of hair removal are the scalp, eyebrows, and eyelashes. Other regions include pubic hair, body hair, and facial hair [3]. Although it is a nonscarring type of hair loss, if hair is continuously pulled, it may no longer grow [4].

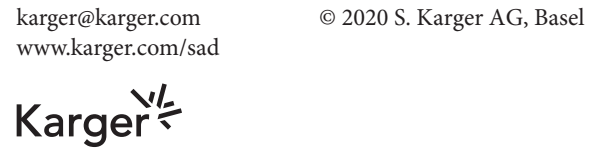


Since 1889, the etiology, epidemiology, natural history, psychosocial impact, and treatment options are still uncertain. TTM onset may occur across the lifespan, yet adolescent onset (ages 10-13) is most frequently reported, affecting more females than males $[5,6]$. The lifetime prevalence is estimated to be around 1-3\% [6]. Nonetheless, there are variations in these findings.

The distressing nature of this disease can lead to severe functional impairment and/or psychological comorbidity. Some patients may suffer from trichophagia, where they consume the pulled hair creating trichobezoars. The trichobezoars, or hairballs, can lead to gastrointestinal difficulties [7]. Psychological comorbidities commonly include mood disorders, anxiety disorders, substance use disorders, eating disorders, and disruptive behavior in youth [8]. There is no definitive cure for TTM in children, with treatment options varying from patient education and behavioral therapy to over-the-counter supplements and medications such as $\mathrm{N}$-acetylcysteine (NAC) or topical therapies like steroids with variable potencies [9].

There has been limited text in pediatric TTM until recently. Studies have claimed that patients initially present at dermatology due to the associated hair loss [9]. Other studies have found patients seek help from a mental health clinician as TTM is considered a psychiatric diagnosis [10]. Both fields of medicine evaluate a noteworthy amount of TTM cases; however, diagnostic measures, management, and treatment of TTM may differ between the two.

Hence, we assessed the clinical and epidemiologic characteristics of this disease in a diverse patient population at our urban tertiary care center. We sought to add to the growing literature with the primary objective of providing families an improved understanding of TTM in children.

\section{Materials and Methods}

\section{Participants and Procedures}

After receiving IRB approval/exemption (Children's Hospital of Philadelphia; IRB 18-015808), the EPIC database was queried 1/1/18-12/31/19 for TTM using ICD-10-CM Code F63.3. Subjects were excluded if initial presentation was at age 18 or over. Age and gender of the subject, growth parameters (height, weight, and BMI), self-described race/ethic background, past medical history, family history, age at first presentation of TTM, location of TTM, chief complaint, clinical characteristics for diagnosis, and treatment/management for TTM were abstracted.
Statistical Analysis

Baseline and demographic characteristics were summarized by standard descriptive methods (e.g., means and standard deviations for continuous variables such as age and numbers with percentages for categorical variables such as gender).

\section{Results}

\section{All Study Participants}

A total of 137 eligible subjects were evaluated. Fortyeight (35.0\%) were males, and 89 (65.0\%) were females. The most common self-reported race and ethnicity were white $(n=90,65.7 \%)$ and non-Hispanic/Latino $(n=124$, $90.5 \%)$, respectively. Average age at diagnosis was $7.93 \pm$ 4.08 years. For initial presentation, $50(36.5 \%)$ were treated by dermatology and 97 (63.5\%) were treated by behavioral health/child development. Eighty-six (62.8\%) had a psychiatric comorbidity, and $32(23.4 \%)$ had a skin disorder. Sixty-five $(57.4 \%)$ had a family history of a psychiatric comorbidity, and $37(27.0 \%)$ had a family history of a skin disorder (shown in Table 1). Subject psychiatric comorbidities included attention deficit hyperactivity disorder (ADHD) $(n=38)$, general anxiety disorder $(n=$ $50)$, tic disorder $(n=11)$, learning/language delays $(n=$ $21)$, depression $(n=4)$, Asperger's $(n=1)$, autism spectrum disorder $(n=10)$, obsessive-compulsive disorder $(n=8)$, behavioral concerns $(n=9)$, suicidal ideation $(n=3)$, sensory stimulation-seeking impulsive disorder $(n=1)$, and psychiatric condition $(n=1)$. Examples of skin disorders the subjects had include eczema $(n=16)$, alopecia areata $(n=6)$, dermatitis $(n=3)$, warts $(n=1)$, neurofibromatosis-1 (NF1) $(n=1)$, vascular malformation $(n=1)$, telogen effluvium $(n=1)$, keratosis pilaris $(n=1)$, psoriasis $(n=1)$, and hemangioma $(n=4)$. Some subjects had more than one psychiatric disorder and/or skin disorder.

Chief complaints included pulling hair $(n=77,56.6 \%)$; hair loss ( $n=18,13.2 \%)$; eating hair $(n=1,0.7 \%)$; pulling hair and hair loss $(n=24,17.6 \%)$; pulling hair and eating hair $(n=13,9.6 \%)$; pulling hair, hair loss, and eating hair $(n=3,2.2 \%)$; and not specified $(n=1,0.7 \%)$ (shown in Table 1). Three female patients had trichobezoars found, of which 2 were treated by dermatology for initial presentation and 1 was treated by behavioral health/child development for initial presentation.

Location of TTM included scalp $(n=96,70.6 \%)$; eyebrows $(n=5,3.7 \%)$; eyelashes $(n=5,3.7 \%)$; body hair ( $n=1,0.7 \%)$; scalp and eyelashes $(n=4,2.9 \%)$; eyebrows and eyelashes ( $n=12,8.8 \%)$; scalp, eyebrows, and eye- 
Table 1. Demographics, past medical history, family history, clinical characteristics of TTM, and treatment in all study participants

\begin{tabular}{|c|c|c|c|}
\hline Descriptor & $\begin{array}{l}\text { Total sample } \\
(n=137)\end{array}$ & Descriptor & $\begin{array}{l}\text { Total sample } \\
(n=137)\end{array}$ \\
\hline Gender & & Telogen effluvium & 1 \\
\hline Female & $89(65.0 \%)$ & Psoriasis & 1 \\
\hline Self-reported race & & Hemangioma & 4 \\
\hline American Indian or Alaska Native & $0(0 \%)$ & $\%$ with family history of psychiatric comorbidity & $65(47.4 \%)$ \\
\hline Black or African American & $21(15.3 \%)$ & Age at diagnosis $($ mean \pm SD) & $7.93 \pm 4.08$ \\
\hline Indian & $1(0.7 \%)$ & Initial presentation specialty & \\
\hline Native Hawaiian or other Pacific Islander & $0(0 \%)$ & Dermatology & $50(36.5 \%)$ \\
\hline White & $90(65.7 \%)$ & Behavioral health/child development & $87(63.5 \%)$ \\
\hline Others & $21(15.3 \%)$ & Chief complaint & \\
\hline Refused & $2(1.5 \%)$ & Pulling hair & $77(56.6 \%)$ \\
\hline$\%$ with psychiatric comorbidity & $86(62.8 \%)$ & Pulling hair, hair loss, and eating hair & $3(2.2 \%)$ \\
\hline ADHD & 38 & Not specified & $1(0.7 \%)$ \\
\hline General anxiety disorder & 50 & Location of TTM & \\
\hline Tic disorder & 11 & Scalp & $96(70.6 \%)$ \\
\hline Learning/language delays & 21 & Eyebrows & $5(3.7 \%)$ \\
\hline Depression & 4 & Eyelashes & $5(3.7 \%)$ \\
\hline Asperger's & 1 & Body hair & $1(0.7 \%)$ \\
\hline Autism spectrum disorder & 10 & Scalp and eyelashes & $4(2.9 \%)$ \\
\hline Obsessive-compulsive disorder & 8 & Eyebrows and eyelashes & $12(8.8 \%)$ \\
\hline Behavioral concerns & 9 & Scalp, eyebrows, and eyelashes & $3(2.2 \%)$ \\
\hline Suicidal ideation & 3 & Eyebrows and body hair & $2(1.5 \%)$ \\
\hline Sensory stimulation-seeking impulsive disorder & 1 & Treatment & \\
\hline
\end{tabular}

ADHD, attention deficit hyperactivity disorder; NF1, neurofibromatosis-1; TTM, trichotillomania; SD, standard deviation.

lashes $(n=3,2.2 \%)$; and eyebrows and body hair $(n=2$, $1.5 \%$ ) (shown in Table 1 ).

Treatment options included education $(n=42,30.7 \%)$, medication $(n=21,15.3 \%)$, behavioral therapy/counseling $(n=66,48.2 \%)$, others $(n=1,0.7 \%)$, and none $(n=7$, $5.1 \%)$ (shown in Table 1). Other treatments included cutting off hair $(n=1)$.

\section{Participants Initially Evaluated by Dermatology}

Of the 137 eligible subjects evaluated, 50 were treated by dermatology at initial presentation. Sixteen (32.0\%) were males, and $34(68.0 \%)$ were females. The most common self-reported race was white $(n=32,64.0 \%)$, and the most common self-reported ethnicity was non-Hispanic/ Latino $(n=45,90.0 \%)$. Average age at diagnosis was 8.29 \pm 4.30 years. Of the 50 subjects, $23(46.0 \%)$ had a psychiatric comorbidity and 19 (38.0\%) had a skin disorder. Psychiatric comorbidities of subjects that presented to dermatology included ADHD $(n=10)$, general anxiety disorder $(n=13)$, learning/language delays $(n=5)$, suicidal ideation $(n=1)$, autism spectrum disorder $(n=1)$, behavioral concerns $(n=4)$, obsessive-compulsive disorder $(n=3)$, sensory stimulation-seeking impulsive disorder $(n=1)$, and psychiatric condition $(n=1)$. Skin disorders of subjects treated by dermatology included alopecia areata $(n=5)$, eczema $(n=12)$, hemangioma $(n=2)$, der- 
Table 2. Demographics, past medical history, family history, clinical characteristics of TTM, and treatment in study participants seen by dermatology

\begin{tabular}{|c|c|}
\hline Descriptor & $\begin{array}{l}\text { Total sample } \\
(n=50)\end{array}$ \\
\hline \multicolumn{2}{|l|}{ Gender } \\
\hline Male & $16(32.0 \%)$ \\
\hline Female & $34(68.0 \%)$ \\
\hline \multicolumn{2}{|l|}{ Self-reported race } \\
\hline American Indian or Alaska native & $0(0 \%)$ \\
\hline Asian & $2(4.0 \%)$ \\
\hline Black or African American & $10(20.0 \%)$ \\
\hline Indian & $0(0 \%)$ \\
\hline Native Hawaiian or other Pacific Islander & $0(0 \%)$ \\
\hline White & $32(64.0 \%)$ \\
\hline Others & $5(10.0 \%)$ \\
\hline Refused & $1(2.0 \%)$ \\
\hline \multicolumn{2}{|l|}{ Self-reported ethnicity } \\
\hline Hispanic or Latino & $4(8.0 \%)$ \\
\hline Non-Hispanic or Latino & $45(90.0 \%)$ \\
\hline Refused & $1(2.0 \%)$ \\
\hline$\%$ with psychiatric comorbidity & $23(46.0 \%)$ \\
\hline ADHD & 10 \\
\hline General anxiety disorder & 14 \\
\hline Learning/language delays & 5 \\
\hline Suicidal ideation & 1 \\
\hline Autism spectrum disorder & 1 \\
\hline Behavioral concerns & 4 \\
\hline Obsessive-compulsive disorder & 3 \\
\hline Sensory stimulation-seeking impulsive disorder & 1 \\
\hline Psychiatric condition & 1 \\
\hline$\%$ with skin disorder & $19(38.0 \%)$ \\
\hline Alopecia areata & 5 \\
\hline Eczema & 12 \\
\hline Hemangioma & 2 \\
\hline Dermatitis & 1 \\
\hline Acne & 3 \\
\hline Vascular malformation & 1 \\
\hline Telogen effluvium & 10 \\
\hline Keratosis pilaris & 1 \\
\hline Psoriasis & 1 \\
\hline$\%$ with family history of psychiatric comorbidity & $15(30.0 \%)$ \\
\hline$\%$ with family history of skin disorder & $21(42.0 \%)$ \\
\hline Age at diagnosis $($ mean $\pm S D)$ & $8.29 \pm 4.30$ \\
\hline \multicolumn{2}{|l|}{ Chief complaint } \\
\hline Pulling hair & $15(30.0 \%)$ \\
\hline Hair loss & $16(32.0 \%)$ \\
\hline Eating hair & $1(2.0 \%)$ \\
\hline Pulling hair and hair loss & $13(26.0 \%)$ \\
\hline Pulling hair and eating hair & $3(6.0 \%)$ \\
\hline Pulling hair, hair loss, and eating hair & $2(4.0 \%)$ \\
\hline \multicolumn{2}{|l|}{ Location of TTM } \\
\hline Scalp & $41(82.0 \%)$ \\
\hline Eyelashes & $2(4.0 \%)$ \\
\hline Scalp and eyelashes & $2(4.0 \%)$ \\
\hline Eyebrows and eyelashes & $4(8.0 \%)$ \\
\hline Scalp, eyebrows, and eyelashes & $1(2.0 \%)$ \\
\hline \multicolumn{2}{|l|}{ Treatment } \\
\hline Education & $17(34.0 \%)$ \\
\hline Medication & $17(34.0 \%)$ \\
\hline Behavioral therapy/counseling & $14(28.0 \%)$ \\
\hline Others & $0(0 \%)$ \\
\hline None & $2(4.0 \%)$ \\
\hline
\end{tabular}

ADHD, attention deficit hyperactivity disorder; TTM, trichotillomania; SD, standard deviation.

matitis $(n=1)$, acne $(n=3)$, vascular malformation $(n=$ $1)$, telogen effluvium $(n=10)$, keratosis pilaris $(n=1)$, and psoriasis $(n=1)$. Some subjects had more than one psychiatric disorder and/or skin disorder. Fifteen (30.0\%) had a family history of a psychiatric comorbidity, and 21
(42.0\%) had a family history of a skin disorder (shown in Table 2).

Chief complaints in the 50 subjects included pulling hair $(n=15,30.0 \%)$; hair loss $(n=16,32.0 \%)$; eating hair $(n=1,2.0 \%)$; pulling hair and hair loss $(n=13,26.0 \%)$; pulling hair and eating hair $(n=3,6.0 \%)$; and pulling hair, hair loss, and eating hair $(n=2,4.0 \%)$ (shown in Table 2).

Location of TTM in the 50 subjects included scalp ( $n=41,82.0 \%)$; eyelashes $(n=2,4.0 \%)$; scalp and eyelashes $(n=2,4.0 \%)$; eyebrows and eyelashes $(n=4,8.0 \%)$; and scalp, eyebrows, and eyelashes $(n=1,2.0 \%)$ (shown in Table 2).

Treatment options in the 50 subjects included education $(n=17,34.0 \%)$, medication $(n=17,34.0 \%)$, behavioral therapy/counseling $(n=14,28.0 \%)$, others $(n=0$, $0 \%)$, and none $(n=2,4.0 \%)$ (shown in Table 2). Medications that dermatologists prescribed included selenium sulfide shampoo $(n=1)$, minoxidil $(n=3)$, topical tofacitinib $(n=1)$, fluocinonide ointment $(n=2)$, clobetasol propionate $(n=3)$, NAC $(n=1)$, pimecrolimus $(n=1)$, hydrocortisone lotion $(n=1)$, fluocinolone solution $(n=$ $3)$, and triamcinolone $(n=1)$.

\section{Participants Initially Evaluated by Behavioral Health/ Child Development}

Of the 137 eligible subjects evaluated, 87 were treated by behavioral health/child development at initial presentation. Thirty-two (36.8\%) were males, and 55 (63.2\%) were females. The most common self-reported race and ethnicity were white $(n=58,66.7 \%)$ and non-Hispanic/ Latino $(n=79,90.8 \%)$, respectively. Average age at diagnosis was $7.72 \pm 3.97$ years. Of the 87 subjects, 63 (72.4\%) had a psychiatric comorbidity and 13 (14.9\%) had a skin disorder. Psychiatric disorders of subjects treated by behavioral health included $\mathrm{ADHD}(n=28)$, general anxiety disorder $(n=37)$, tic disorder $(n=11)$, learning/language delays $(n=16)$, depression $(n=4)$, Asperger's $(n=1)$, suicidal ideation $(n=2)$, autism spectrum disorder $(n=$ 9 ), behavioral concerns $(n=5)$, and obsessive-compulsive disorder $(n=5)$. Skin disorders of subjects that presented to behavioral health included alopecia areata $(n=1)$, eczema $(n=4)$, hemangioma $(n=2)$, dermatitis $(n=2)$, warts $(n=1)$, acne $(n=2)$, and NF1 $(n=1)$. Some subjects had more than one psychiatric disorder and/or skin disorder. Fifty (57.5\%) had a family history of a psychiatric comorbidity, and $16(18.4 \%)$ had a family history of a skin disorder (shown in Table 3).

Chief complaints in the 87 subjects included pulling hair $(n=62,72.1 \%)$; hair loss $(n=2,2.3 \%)$; pulling hair 
Table 3. Demographics, past medical history, family history, clinical characteristics of TTM, and treatment in study participants seen by behavioral health/child development

\begin{tabular}{|c|c|}
\hline Descriptor & $\begin{array}{l}\text { Total sample } \\
(n=87)\end{array}$ \\
\hline \multicolumn{2}{|l|}{ Gender } \\
\hline Male & $32(36.8 \%)$ \\
\hline Female & $55(63.2 \%)$ \\
\hline \multicolumn{2}{|l|}{ Self-reported race } \\
\hline American Indian or Alaska Native & $0(0 \%)$ \\
\hline Asian & $0(0 \%)$ \\
\hline Black or African American & $11(12.6 \%)$ \\
\hline Indian & $1(1.1 \%)$ \\
\hline Native Hawaiian or other Pacific Islander & $0(0 \%)$ \\
\hline White & $58(66.7 \%)$ \\
\hline Others & $16(18.4 \%)$ \\
\hline Refused & $1(1.1 \%)$ \\
\hline \multicolumn{2}{|l|}{ Self-reported ethnicity } \\
\hline Hispanic or Latino & $7(8.0 \%)$ \\
\hline Non-Hispanic or Latino & $79(90.8 \%)$ \\
\hline Refused & $1(1.1 \%)$ \\
\hline$\%$ with psychiatric comorbidity & $63(72.4 \%)$ \\
\hline ADHD & 28 \\
\hline General anxiety disorder & 37 \\
\hline Tic disorder & 11 \\
\hline Learning/language delays & 16 \\
\hline Depression & 4 \\
\hline Asperger's & 1 \\
\hline Suicidal ideation & 2 \\
\hline Autism spectrum disorder & 9 \\
\hline Behavioral concerns & 5 \\
\hline Obsessive-compulsive disorder & 5 \\
\hline$\%$ with skin disorder & $13(14.9 \%)$ \\
\hline Alopecia areata & 1 \\
\hline Eczema & 4 \\
\hline Hemangioma & 2 \\
\hline Dermatitis & 2 \\
\hline Warts & 1 \\
\hline Acne & 2 \\
\hline NF1 & 1 \\
\hline$\%$ with family history of psychiatric comorbidity & $50(57.5 \%)$ \\
\hline$\%$ with family history of skin disorder & $16(18.4 \%)$ \\
\hline Age at diagnosis (mean $\pm \mathrm{SD})$ & $7.72 \pm 3.97$ \\
\hline \multicolumn{2}{|l|}{ Chief complaint } \\
\hline Pulling hair & $62(72.1 \%)$ \\
\hline Hair loss & $2(2.3 \%)$ \\
\hline Pulling hair and hair loss & $11(12.8 \%)$ \\
\hline Pulling hair and eating hair & $10(11.6 \%)$ \\
\hline Pulling hair, hair loss, and eating hair & $1(1.2 \%)$ \\
\hline \multicolumn{2}{|l|}{ Location of TTM } \\
\hline Scalp & $55(64.0 \%)$ \\
\hline Eyebrows & $5(5.8 \%)$ \\
\hline Eyelashes & $11(12.8 \%)$ \\
\hline Body hair & $1(1.2 \%)$ \\
\hline Scalp and eyelashes & $2(2.3 \%)$ \\
\hline Eyebrows and eyelashes & $8(9.3 \%)$ \\
\hline Scalp, eyebrows, and eyelashes & $2(2.3 \%)$ \\
\hline Eyebrows and body hair & $2(2.3 \%)$ \\
\hline \multicolumn{2}{|l|}{ Treatment } \\
\hline Education & $25(28.7 \%)$ \\
\hline Medication & $4(4.6 \%)$ \\
\hline Behavioral therapy/counseling & $52(59.8 \%)$ \\
\hline Others & $1(1.2 \%)$ \\
\hline None & $5(5.7 \%)$ \\
\hline
\end{tabular}

ADHD, attention deficit hyperactivity disorder; NF1, neurofibromatosis-1; TTM, trichotillomania; $\mathrm{SD}$, standard deviation

and hair loss ( $n=11,12.8 \%)$; pulling hair and eating hair ( $n=10,11.6 \%)$; and pulling hair, hair loss, and eating hair $(n=1,1.2 \%)$ (shown in Table 3 ).

Location of TTM in the 87 subjects included scalp ( $n=55,64.0 \%)$; eyebrows $(n=5,5.8 \%)$; eyelashes $(n=11$,
$12.8 \%)$; body hair $(n=1,1.2 \%)$; scalp and eyelashes $(n=$ $2,2.3 \%)$; eyebrows and eyelashes $(n=8,9.3 \%)$; scalp, eyebrows, and eyelashes $(n=2,2.3 \%)$; and eyebrows and body hair $(n=2,2.3 \%)$ (shown in Table 3 ).

Treatment options in the 87 subjects included education $(n=25,28.7 \%)$, medication $(n=4,4.6 \%)$, behavioral therapy/counseling $(n=52,59.8 \%)$, others $(n=1$, $1.2 \%)$, and none ( $n=5,5.7 \%)$ (shown in Table 3). Medications that were prescribed by behavioral health specialists include atomoxetine $(n=1)$, guanfacine $(n=1)$, lisdexamfetamine $(n=1)$, and fluoxetine $(n=1)$.

\section{Discussion/Conclusion}

In this study, we present data on the clinical and epidemiologic features of TTM in a sample of children at an urban tertiary care center. Most subjects in our cohort were females (65\%), keeping with the existing literature $[5,6]$. The average age at initial diagnosis was 7.93. This is contrary to current studies which have reported ages of 10-13 years at initial TTM diagnosis [5]. Our conflicting finding could be due to the fact that parents/guardians were seeking care at a children's hospital rather than a general practice or hospital.

Previous text has suggested that patients initially present for hair loss at dermatology [9]. However, most of our patients were treated by behavioral health at initial presentation (63.5\%). Since patients had more psychiatric comorbidities (62.8\%) than skin disorders (23.4\%), seeking care from behavioral health might have been the most timely and cost-efficient route. Almost half had a family history of psychiatric comorbidities (47.4\%), with only about a quarter with a family history of skin comorbidities $(27.0 \%)$. With over half of our patients having psychiatric comorbidities themselves, it speaks to the possible interplay and effects of this disease on other psychological disorders [8].

The most common locations of TTM were consistent with previous studies, the scalp (70.6\%) followed by the eyebrows and eyelashes in conjunction (8.8\%) [3]. The most common chief complaint was pulling hair alone (56.6\%), emphasizing the compulsive hair pulling nature of TTM. There were 17 total trichophagia cases $(n=1$ just eating hair; $n=16$ combination of trichophagia in conjunction with another chief complaint). This is far greater than the 2 trichophagia cases found in a study done in Croatia [11]. Of the 17 trichophagia cases, 3 female patients had trichobezoars found. Cases of trichobezoars are most commonly reported in females [12]. Those families 
that note trichophagia in their children should be aware of the accompanying complications such as abdominal and epigastric pain, ulcerations, gastric mucosal erosion, and, in severe cases, death [13].

Most families/patients proceeded with cognitive behavioral therapy (CBTI)/counseling (48.2\%). This could be because most presented to behavioral health for evaluation. The next common treatment options were education $(30.7 \%)$ and then medication (15.3\%). There are no pharmacologic cures for TTM; thus, the prescribing rate of medications for TTM is fairly low [14]. Currently, the first-line therapeutic option is habit reversal therapy, suggesting CBTI as a safe and effective therapy [15]. However, large clinical studies must be conducted to determine definitive outcomes in treatment for children with TTM.

Characteristics between patients evaluated by dermatology and behavioral health varied. In dermatology, over a third had a skin disorder $(38.0 \%)$, which is more than the total group percentage that had a skin disorder (23.4\%). In behavioral health, only $14.9 \% \mathrm{had}$ a skin disorder, while almost three-quarters (72.4\%) had a psychiatric comorbidity. Thus, patients may be more likely to present to a specialist that is familiar with their additional health conditions. Hair loss (32.0\%) was the most common chief complaint in dermatology, while pulling hair (72.1\%) was the common chief complaint in behavioral health. Only $2 \%$ of those presenting to behavioral health complained about hair loss alone. This is expected as hair loss is a common disorder treated by pediatric dermatologists [16]. Many families that present to dermatology may be unaware of the compulsive hair pulling and seek treatment for the apparent hair loss instead [17]. On the other hand, families that present to behavioral health are more likely aware of the compulsive hair pulling and seek treatment to decrease this repetitive behavior [18]. In terms of treatment, education $(34.0 \%)$ and medication $(34.0 \%)$ were most common in dermatology, while behavioral therapy (59.8\%) was the most common in behavioral health. Only $4.6 \%$ of those evaluated by behavioral health were recommended medications. Perhaps practicing dermatologists are more likely to address hair loss concerns by prescribing medications for TTM.

This is the first study to look at TTM in those assessed by different fields of medicine, dermatology, and behavioral health. Our results highlight the complex and multifactorial nature of TTM. There were clear differences between the total group findings and findings from those evaluated by dermatology or behavioral health. Young patients are severely affected by TTM, both physically and emotionally; thus, early diagnosis and intervention is key to prevent further exacerbation. The field of psychodermatology is already receiving attention and growing due to the challenges of treating psychological diseases in dermatology [19]. In fact, 12 patients treated in a psychodermatology service for various skin disorders noted improvement in their conditions [20]. Since habit reversal therapy is the most effective therapeutic option to date, dermatologists are recommended to create partnerships with experienced community mental health providers [9].

Although TTM is a psychiatric diagnosis, our study confirms that many see it as a dermatological issue as well because of associated hair loss. With over half of our patients affected by other psychiatric disorders, an interconnection between dermatology and behavioral therapy experts is highly encouraged. Both professionals can offer varying expertise to create a multidisciplinary management plan that is personalized to the individual affected by TTM. These important clinical findings increase the need for supplementary research on the pathology and management of TTM in children.

\section{Limitations}

Our study limitations include its retrospective design and small sample size. To better identify characteristics of the disease and effect and severity of comorbidities, prospective longitudinal cross-sectional studies with a larger sample are needed.

\section{Statement of Ethics}

This study was approved and granted exemption by the Children's Hospital of Philadelphia Institutional Review Board (IRB 18-015808).

\section{Conflict of Interest Statement}

The authors have no conflicts of interest to declare.

\section{Funding Sources}

The authors did not receive any funding.
Rangu/Castelo-Soccio 


\section{Author Contributions}

Dr. Castelo and Ms. Rangu had full access to all of the data in the study and take responsibility for the integrity of the data and the accuracy of the data analysis. Dr. Castelo was responsible for study concept and design, critical revision of the manuscript for important intellectual content, and study supervision. Rangu performed statistical/data analysis. Rangu and Castelo were involved in acquisition, analysis, and interpretation of data and drafting of the manuscript and provided administrative, technical, or material support.

\section{References}

1 Hallopeau M. Alopecie par grattage (trichomanie ou trichotillomanie). Ann Dermatol Syphil. 1889;10:440-1.

2 Substance Abuse and Mental Health Services Administration. Impact of the DSM-IV to DSM-5 changes on the National Survey on Drug Use and Health [Internet]. Rockville (MD): Substance Abuse and Mental Health Services Administration (US) [cited 2016 June]. Table 3.27, DSM-IV to DSM-5 Trichotillomania (Hair-Pulling Disorder) Comparison. Available from: https://www.ncbi.nlm. nih.gov/books/NBK519704/table/ch3.t27/

3 O'Sullivan RL, Keuthen NJ, Christenson GA. Trichotillomania: behavioral symptom or clinical syndrome? Am J Psychiatry. 1997; 154:1442-9.

4 Sperling LC, Mezebish DS. Hair diseases. Med Clin North Am. 1998;82(5):1155-69.

5 Ricketts EJ, Snorrason I, Kircanski K, Alexander JR, Stiede JT, Thamrin H, et al. A latent profile analysis of age of onset in trichotillomania. Ann Clin Psychiatry. 2019 Aug;31(3): 169-78.

6 Christenson GA, Pyle RL, Mitchell JE. Estimated lifetime prevalence of trichotillomania in college students. J Clin Psychiatry. 1991; 52(10):415-7.
7 Bouwer C, Stein DJ. Trichobezoars in trichotillomania: case report and literature overview. Psychosom Med. 1998;60(5):658-60.

8 Harrison JP, Franklin ME. Pediatric trichotillomania. Curr Psychiatry Rep. 2012;14(3): 188-96.

9 Henkel ED, Jaquez SD, Diaz LZ. Pediatric trichotillomania: review of management. Pediatr Dermatol. 2019 Nov;36(6):803-7.

10 Woods DW, Flessner CA, Franklin ME, Keuthen NJ, Goodwin RD, Stein DJ, et al. The trichotillomania impact project (TIP): exploring phenomenology, functional impairment, and treatment utilization. J Clin Psychiatry. 2006;67(12):1877-88.

11 Klobucar A, Folnegovic-Smalc V, Folnegovic-Smalc V, Kocijan-Hercigonja D, Sovic S, Gulic L. Clinical characteristics and comorbidity of pediatric trichotillomania: the study of 38 cases in Croatia. Psychiat Danub. 2018; 30(1):79-84.

12 Antunes H, Barroso C, Faria C, Correia-Pinto J. Images in paediatrics: Rapunzel syndrome: the pathway for a prompt diagnosis. Arch Dis Child. 2020 Mar;105(3):298.

13 Kinoo SM, Singh B. Gastric trichobezoar: an enduring intrigue. Case Rep Gastrointest Med. 2012;2012:136963.
14 Farhat LC, Olfson E, Levine JLS, Li F, Franklin $\mathrm{ME}$, Lee HJ, et al. Measuring treatment response in pediatric trichotillomania: a metaanalysis of clinical trials. J Child Adolesc Psychopharmacol. 2019 Dec 2;30(5):306-15.

15 Association for Behavioral and Cognitive Therapies [Internet]. ABCT Trichotillomania Fact Sheet [cited 2020]. Available from http:// www.abct.org/docs/factsheets/TRICHOTILLOMANIA.pdf.

16 Castelo-Soccio L, McMahon P. Pediatric dermatology. J Clin Aesthet Dermatol. 2017 Mar; 10(3):S8-15.

17 Chandran NS, Novak J, Iorizzo M, Grimalt R, Oranje AP. Trichotillomania in children. Skin Appendage Disord. 2015;1(1):18-24.

18 Flessner CA. Cognitive-behavioral therapy for childhood repetitive behavior disorders: tic disorders and trichotillomania. Child Adolesc Psychiatr Clin N Am. 2011;20(2):31928.

19 Tracey EH. Challenges of treating primary psychiatric disease in dermatology. Cutis. 2019 Aug;104(2):E12-14.

20 Carr JM, Mortimer H, Martin K, Kaur M, Goulding JMR. A retrospective review of 12 patients with trichotillomania treated in a psychodermatology service. Clin Exp Dermatol. 2019 Aug;44(6):658-60. 